\title{
Programa de educación para la salud: Estilos de vida y factores de riesgos en universitarios
}

\section{Health program: Lifestyle and risk factors in college students}

BOJORQUEZ-DIAZ, Cecilia Ivonne, REYNOSO-ERAZO, Leonardo, FAVELA-RAMIREZ, Carlos Artemio y CASTRO-ROBLES, Alejandra Isabel

Instituto Tecnológico de Sonora

ID $1^{\text {er }}$ Autor: Cecilia Ivonne, Bojorquez-Diaz / ORC ID: 0000-0003-0237-5079, CVU CONACYT ID: 279125

ID $1{ }^{\text {er }}$ Coautor: Leonardo, Reynoso-Erazo / ORC ID: 0000-0001-9793-1431, CVU CONACYT ID: 230387

ID $2^{\text {do }}$ Coautor: Carlos Artemio, Favela-Ramirez / ORC ID: 0000-0002-0648-5673, CVU CONACYT ID: 983306

ID $3^{\text {er }}$ Coautor: Alejandra Isabel, Castro-Robles / ORC ID: 0000-0001-7651-5615, CVU CONACYT ID: 1015540

DOI: $10.35429 / J U M .2019 .7 .3 .1 .8$

Recibido 12 de Enero, 2019; Aceptado 30 de Marzo, 2019

\begin{abstract}
Resumen
El propósito de la investigación, fue determinar la eficacia de un programa educativo para cambio de estilos de vida en relación a factores de riesgo en sujetos con la enfermedad y/o en riesgos de padecer alguna o ambas de las enfermedades crónico degenerativas, implementado en una universidad de México. Se trabajó con 40 universitarios entre 18 y 20 años. El diseño del estudio fue descriptivo, transversal, con mediciones antes y después de la intervención. Los resultados arrojaron incidencia de hipertensión arterial alta para la sistólica de $100 \%$, siendo estadísticamente significativo. Al evaluar la eficacia del programa en cuanto al IMC y conocimiento, Los resultados no fueron significativos. Sin embargo, al realizar el análisis de forma individual, si se presentaron cambios en el conocimiento, reflejado en el aumento de las medias; y en el mismo sentido, hubo también decremento de forma individual en el IMC. Aun cuando el programa no muestra estadísticas significativas, tuvo impacto en el $73 \%$ de la muestra. La investigación aplicada es generadora de aprendizaje, ya que, en este caso, al diseñar, aplicar y evaluar el programa, da oportunidad de corregir estrategias y procedimientos que ayuden a disminuir los factores de riesgo y mejorar la salud del universitario.
\end{abstract}

Educación, Salud, Factores de Riesgo

\begin{abstract}
The purpose of the study was to determine the efficacy of an educational program for change lifestyles related to risk factors on subjects with a disease or in the risk of suffering one or both chronic-degenerative diseases, implemented at a university in northwestern Mexico. A sample of 40 college students between 18 and 20 years was studied, indicating inclusión criteria. This study used a descriptive transversal design, with measurements before and after the intervention. Results showed hypertension incidence on elevated systolic pressure in $100 \%$ of cases, being statistically significative. Regarding the evaluation of program effectiveness on the Body Mass Index (BMI) and knowledge, statistically, no differences were found. However, within individual-analysis, reflected knowledge showed an increase; in the same way, there was an individual decrement on BMI. Even when the program does not show significative differences, had an impact on $73 \%$ of the sample. Applicated research it is a learning generator, thus, in this case, at the moment of design, apply, and evaluate the program, give us the opportunity to correct strategies and procedures that help to decrease risk factors and improves the college student health.
\end{abstract}

Citación: BOJORQUEZ-DIAZ, Cecilia Ivonne, REYNOSO-ERAZO, Leonardo, FAVELA-RAMIREZ, Carlos Artemio y CASTRO-ROBLES, Alejandra Isabel. Programa de educación para la salud: Estilos de vida y factores de riesgos en universitarios. Revista de Gestión Universitaria. 2019. 3-7: 1-8

*Correspondencia del Autor (correo electrónico: cecy_884@ hotmail.com)

$\dagger$ Investigador contribuyendo como primer autor. 


\section{Introducción}

El presente estudio aborda la problemática de las enfermedades crónicas no transmisibles (ECNT) como la hipertensión y diabetes mellitus tipo 2 . Ya que la Organización Mundial de la Salud (O.M.S.) menciona que actualmente se han vinculado a los estilos de vida inadecuados que hoy llevan los individuos (OMS, 2018). El aparecimiento de las enfermedades crónico no transmisibles como la hipertensión y diabetes en la actualidad, no pueden ignorarse. Se calculaba que aproximadamente 30 millones de personas en el mundo padecían diabetes en 1985. Para el año de 1995 la cifra se había incrementado a 135 millones y la estimación de la OMS para el número total de diabéticos era de 177 millones de personas en el año 2000, de los cuales más de 2 millones son de México. Se pronostica que la cifra se incrementará hasta 300 millones para el año 2025, de los cuales más de 6 millones serán mexicanos, y las principales causas o factores de riesgo con los que se asocian estas enfermedades son los estilos de vida inadecuados (OMS, 2013).

De acuerdo a estadísticas presentadas por el Instituto Nacional de Estadística Geografía e información (INEGI) en el periodo de 19902008 para el estado de Sonora, reporta a la Hipertensión arterial como una de las principales causas de muerte que afectan a los diferentes grupos de edad con el 81.64, siguiendo la diabetes mellitus con 61.9 casos, lo anterior por cada 100 mil habitantes por período anual (INEGI, 2010).

Con base a lo anterior y los resultados obtenidos de estudios previos en la universidad en los cuales se refleja ampliamente la presencia de altos índices de obesidad, sedentarismo, estrés, y herencia, se plantea realizar un análisis sobre el estilo de vida de los estudiantes de una universidad de Sonora México, que presenten factores de riesgos asociados con las enfermedades crónico no transmisibles.

La aportación de este trabajo es pasar de la investigación básica a la aplicada. Es decir, de la exploración y descripción a la intervención, y así poder incidir en los estilos de vida de los jóvenes.
Para esto, se diseñó, implementó y se evaluó la eficacia de un programa educativo para cambio de estilos de vida en relación a factores de riesgo en sujetos con la enfermedad y/o en riesgos de padecer alguna o ambas de las enfermedades crónico-degenerativas, en estudiantes universitarios.

El programa se elaboró apoyado en el modelo educación para la salud, ya que abarca más que los enfoques prescriptivos y conductuales basados en aspectos sanitarios. Este modelo representa una propuesta participativa, holística y de transversalidad en el funcionamiento institucional y pedagógico de las organizaciones escolares, empoderando las actitudes y comportamientos de las personas como responsables de su propia salud (Chamorro, 2010). Este tipo de programas han reportado casos de éxito en diferentes países. Uno de los más actuales es el de Wolferz, Arjani, Bolze, y Frates (2019) en el cual demuestran la eficacia de la educación en los estilos de vida, a través de lograr que los jóvenes se responsabilicen de su propia salud.

\section{Objetivo}

Promover a través del programa de intervención, hábitos de vida saludable y capacitación para identificar los factores de riesgo que pueden provocar enfermedades como la hipertensión y diabetes mellitus tipo 2 .

Los objetivos específicos fueron, (1) conocer la incidencia y/o prevalencia de tensión arterial y de hiperglucemia, y en qué medida se relacionan con los factores de riesgo de obesidad, actividad física, herencia, sedentarismo y estrés. (2) cuál será la eficacia en cuanto a conocimiento del programa educativo implementado para el cambio de estilos de vida, con los factores de riesgo para controlar a las personas que padecen enfermedades crónicodegenerativas y los que están en riesgo de desarrollar la enfermedad. (3) en qué medida el ofrecer alternativas educativas de alimentación, actividad física y manejo del estrés puede impactar en el índice de masa corporal de la población sujeto de estudio

\section{Método}

Diseño. El diseño del estudio fue descriptivo, transversal, con mediciones antes y después de la intervención (Hernández, Fernández y Baptista, 2016).

BOJORQUEZ-DIAZ, Cecilia Ivonne, REYNOSO-ERAZO, Leonardo, FAVELA-RAMIREZ, Carlos Artemio y CASTRO-ROBLES, Alejandra Isabel. Programa de educación para la salud: Estilos de vida y factores de riesgos en universitarios. Revista de Gestión Universitaria. 2019 
Participantes. Para desarrollar la presente investigación se trabajó con una muestra de conveniencia, no paramétrica con un grupo natural de 40 estudiantes, tomando en cuenta los siguientes criterios de inclusión. Alumnos correspondientes al segundo semestre, sin importar el género, mayores de 18 años, solteros, que presenten hipertensión y/o diabetes o cualquier factor de riesgo como: sobrepeso, obesidad, sedentarismo, herencia y que no realicen ningún tipo de actividad física o deporte.

\section{Instrumentos}

Se utilizaron dos instrumentos los cuales se describen a continuación: cuestionario de factores de riesgo (FR). El instrumento está conformado por 135 ítems. Contiene preguntas dicotómicas que investiga sobre antecedentes hereditarios y familiares, sobre diabetes, cáncer y obesidad; así como antecedentes de enfermedad cardiovascular.

Otro de los apartados recaba información acerca de los hábitos alimenticios, compuesta por preguntas dicotómicas y de frecuencia; al igual que la escala de actividad física. Siguiendo a estas escalas están la de diversiones, sueño y salud, las cuales se contestan de la misma forma que las anteriores. El alfa de cronbach fue de 0.783 en una aplicación con 181 sujetos realizada por Pancich, Toledo, Monrroy y Reynoso-Erazo (2011). Es similar al utilizado en estudios previos por García et al (2009) y por Camacho et al. (2010).

El segundo instrumento fue un cuestionario de conocimiento sobre estilos de vida y factores de riesgo en estudiantes universitarios. Se elaboró un cuestionario con el objetivo de medir el conocimiento y aprendizaje del programa de educación para la salud; el cual se utilizará como pre-prueba y pos-prueba para evaluar la efectividad del programa aplicado. Está compuesto por 17 ítems con respuestas de opción múltiple. El instrumento fue sometido al procedimiento de validación por parte de cuatro expertos en la materia.

\section{Procedimiento}

Una vez seleccionada la muestra, según los criterios establecidos para la investigación, se citó a los estudiantes a una reunión en la cual se les explicó el objetivo del estudio.
Al finalizar la explicación se les entregó un sobre cerrado, con el consentimiento informado, y se les resaltó que la participación era completamente voluntaria y confidencial. Seguido a lo anterior el investigador contactó a los estudiantes que aceptaron participar en el estudio, estableciendo día y la hora para una reunión, con el objetivo de establecer el encuadre del programa de intervención, y se fijó el horario para llevar a cabo la implementación del trabajo.

La intervención del programa educativo para la salud sobre estilos de vida y factores de riesgos en estudiantes universitarios constó de 16 horas. La primera actividad fue, la aplicación del cuestionario de conocimiento (pre-test). Seguido de la revisión de todos los temas que se verían a lo largo del curso de forma general. Además, se abordó específicamente el tema de hipertensión arterial y diabetes para conocer su etiología. Los dos temas se expusieron, para que conocieran cuando se denomina presión arterial alta o baja, y además que aprendieran a identificar la diferencia entre diabetes mellitus y diabetes mellitus tipo 2 .

En otra actividad se presentó el tema de las enfermedades crónico no transmisibles y factores de riesgo como: obesidad, sedentarismo, estrés y actividad física. Posteriormente se abordó de forma específica los temas de obesidad, sedentarismo, actividad física y estrés.

Se presentó el tema de nutrición: el plato del bien comer, la jarra del buen beber, tabla de equivalencia de alimentos y estrategias para una alimentación saludable. En otra de las actividades se abordaron las diferencias entre ejercicio, actividad física y sedentarismo. Se trabajó con una presentación sobre los temas de estrés, afrontamiento y, solución de problemas, continuando con el tema de estilo de vida saludable. Por último, se llevó a cabo la aplicación del cuestionario de conocimiento (pos-test).

Análisis de datos. Una vez obtenida la información, se procedió a codificarlos en una base de datos, utilizando el paquete estadístico Statistical Package for the Social Sciences (SPSS 21). Se realizaron análisis de frecuencias y medias. Para comparar los resultados de las mediciones entre pre-test y pos-test se aplicó la prueba $t$ para muestras independientes. 
Con lo anterior se establecieron las medidas de tendencia central, dispersión y relación entre variables.

\section{Resultados}

Respecto al primer objetivo específico: conocer la incidencia y/o prevalencia de tensión arterial y de hiperglucemia, y en qué medida se relacionan con los factores de riesgo de obesidad, actividad física, herencia, sedentarismo y estrés. Se obtuvo la información de 40 estudiantes universitarios referente a sus antecedentes hereditarios sobre hipertensión arterial y diabetes, así como datos específicos sobre el estilo de vida que llevan en cuanto a actividad física, sedentarismo, alimentación y estrés.

Aunado a lo anterior se contó con el peso y las cifras de presión arterial del participante. Se reportaron 35 mujeres y cinco hombres con edades entre 18 y 21 años de edad, estableciendo 19.15 como la media. Se describe, que no se presentó ningún caso de diabetes mellitus tipo 2 entre los estudiantes. En la Tabla 1 con respecto a la hipertensión arterial se observa, que se presentó un valor elevado en el $100 \%$ de la muestra participante, presentando una cifra sistólica de $132 \mathrm{~mm} \mathrm{Hg}$ en mujeres y $152 \mathrm{~mm}$ $\mathrm{Hg}$ en hombres; y una cifra en la diastólica de 86.14 en el sexo femenino y 107.60 en el sexo masculino; dicha diferencia que se presenta entre hombres y mujeres fue estadísticamente significativa, aclarando que hubo un sesgo hacia el sexo femenino $(p=.042$ y .002 respectivamente).

Para contrastar la hipertensión arterial con la obesidad, en primera instancia se calculó el índice de masa corporal (IMC) del cual se obtuvo que 25 de los participantes mostraron sobrepeso u obesidad, y 14 reportaron peso normal. Faltó un sujeto de reportar dado que no se encontró la estatura.

Una vez obtenido el índice de masa corporal, se establece que el valor mayor a $25 \mathrm{se}$ considera sobre peso y un valor igual o mayor a 30 se considera obesidad. Con lo anterior, se procedió a comparar por grupo, el nivel de hipertensión arterial, encontrándose una diferencia estadísticamente significativa en el puntaje de la sistólica $(p=.022)$ en donde el grupo con sobrepeso y obesidad presentaron un nivel más alto de tensión arterial $(140.57 \mathrm{~mm}$ $\mathrm{Hg}$ ).
La variable de actividad física considerada como el ejercicio que se recomienda que una persona realice, y que impacte en un estilo de vida saludable, es de tres veces por semana, con una duración de 60 minutos. Pero en los resultados se reportó, que solo el $18 \%$ de la muestra lo realiza.

La variable estrés se midió con los ítems relacionados con las horas que duerme y si este es continuo o en periodos. A lo cual 15 participantes reportan dormir menos de 6 horas, pero 34 mencionaron dormir de manera continua. Además, se tomó en cuenta la pregunta de si al levantarse se sentían descansados y con energía, y solo seis estudiantes mencionaron que siempre.

En repuesta al objetivo específico número dos: cuál será la eficacia en cuanto a conocimiento del programa educativo implementado para el cambio de estilos de vida, con los factores de riesgo para controlar a las personas que padecen enfermedades crónicodegenerativas y los que están en riesgo de desarrollar la enfermedad. Se realizaron análisis de frecuencia, descriptivos y medidas de tendencia central. Los resultados obtenidos muestran la descripción de cada pregunta realizada en el cuestionario de conocimiento, mostrando los puntajes obtenidos del pre test y pos test de la aplicación del programa. Uno de los datos relevantes, estuvo en el ítem número trece, el cual preguntaba sobre el significado de dieta.

La diferencia entre el pre test y el pos test obtuvo una media aritmética de 3.0. Otro puntaje importante en el cual se refleja la incorporación del conocimiento en los estudiantes, corresponde a los beneficios de realizar actividad física con una media de 2.95. Por último, se destaca el aumento de conocimiento, relacionado con los factores de riesgo y su relación con las enfermedades crónico no transmisible, con una media de 5.073 refiriendo específicamente la hipertensión arterial y diabetes mellitus tipo 2, variables centrales en este estudio.

Dentro del cuestionario de conocimiento sobre estilos de vida y factores de riesgo en estudiantes universitarios, se analizó una pregunta que se hacía, sobre la disposición de participar en un programa integral, con especialistas para modificar estilos de vida inadecuados. 
Por un estilo de vida saludable que les permitiera mejorar su salud y por ende su calidad de vida. Dicha pregunta tenía la opción de responder: No estoy dispuesto, me gustaría intentarlo y definitivamente si participaría. A lo anterior el 100 por ciento de la muestra contestó, que definitivamente si participarían, refiriendo como principal motivo, mejorar su calidad de vida.

Los resultados referentes al objetivo específico tres: en qué medida el ofrecer alternativas educativas de alimentación, actividad física y manejo del estrés puede impactar en el índice de masa corporal de la población sujeto de studio

Previo al reporte del IMC se realizó la comparación del peso de los 40 participantes antes del programa, obteniendo una media de 70.305 y después de la intervención, con una media de 69.298.

Los análisis descriptivos arrojaron, que 16 estudiantes tuvieron un decremento menor (de 100 a 200 gramos) en su peso. Cuatro de los participantes bajaron entre 2 y 3.5 kilos y 9 se mantuvieron en su peso. Es decir, el 73 por ciento de la muestra tuvo un comportamiento favorable dentro del estudio. Es importante mencionar que no todos los participantes presentaban sobre peso u obesidad.

Se hicieron comparaciones sobre los resultados obtenidos de las mediciones entre pretest y pos-test, para poder establecer, si el ofrecer alternativas educativas de alimentación, actividad física y manejo del estrés impactó en el índice de masa corporal de la población.

Se presentó una disminución de las medias entre el índice de masa corporal inicial (3.297) contra la medición final (3.194). Para complementar esta información, se realizó un análisis descriptivo por sujeto, y se obtuvo que 18 de los estudiantes que participaron en el estudio, estuvieron por debajo del percentil 25, lo cual significa que pertenecen al rango de peso normal. Uno de los participantes modificó su índice de masa corporal, bajando del percentil 25 al percentil 22, por lo que resalta un gran decremento en su estado, pasando de sobrepeso al peso normal o deseado. Por último, del resto de la muestra se obtuvo que unos se sostuvieron en su mismo índice de masa corporal y otros subieron pocos gramos, pero los hizo subir de percentil.

\section{Discusión}

De los resultados obtenidos se encontró diferencia estadísticamente significativa sobre la incidencia de tensión arterial en la cifra que corresponde a la sistólica, 132 para mujeres y 152 para los hombres. Es decir, el $100 \%$ de la muestra presentó cifras de tensión arterial sistólica por arriba del percentil 120, el cual es considerado como tensión arterial normal. Esto cobra sentido con lo reportado por INEGI (2010) donde proyecta que la hipertensión arterial en el estado de Sonora será una de las principales causas de muerte.

El 64\% de los participantes presentaron sobre peso (18) u obesidad (7), y al hacer la comparación del peso con le tensión arterial, nuevamente esta población reportó niveles más altos de presión arterial sistólica, a diferencia de los de peso normal. El sobrepeso en estas cifras no se considera enfermedad, pero si es una predisposición a padecer enfermedades crónico no transmisibles.

En cambio, la obesidad si representa un factor de riesgo importante para la incidencia y prevalencia de la tensión arterial. Estos resultados coinciden con los hallazgos que señalan Whatnall, Patterson, Brookman, Convery, Swan, Pease, y Hutchesson, (2019) en su estudio sobre comportamientos de estilos de vida y factores de riesgo de salud en una muestra de universitarios australianos, refiriendo que el $39.6 \%$ de 3.077 estudiantes presentaron sobre peso u obesidad, concluyendo que los factores de riesgo y las conductas de estilos de vida poco saludables son altas en su población, por lo que sugieren la implementación de estrategias en pro de la salud de los universitarios.

En cuanto a actividad física y sedentarismo el 66\% de los participantes refieren hacer ejercicio a veces. En este caso el a veces no es suficiente dado que lo recomendado es hacer al menos una hora 3 veces por semana. El plan de acción que propone la OMS (2015) al respecto, menciona la actividad física como una de las estrategias que pueden contribuir para el 2025, a disminuir las enfermedades crónicas no transmisibles y una relación relativa a la mortalidad prematura de un $25 \%$ relacionado también con los índices de obesidad. 
La inconsistente actividad física de esta población, no difiere de los resultados de una muestra de universitarios chinos presentados por Liang, Duan, Shang, Wang, Hu y Lippke, (2019) después de la intervención de un programa de estilos de vida en el cual la activación física era uno de sus objetivos, que al final no obtuvo diferencia significativa, ya que los datos indicaron una alta prevalencia de estilos de vida poco saludables. Sin embargo, refuerzan la importancia de seguir implementando programas que promuevan el cambio del comportamiento en la salud.

Que los estudiantes duerman pocas horas se convierte en un factor de riesgo que puede favorecer el aparecimiento del estrés. El dormir pocas horas trae como consecuencia levantarse cansados y con poca energía. Este patrón de sueño se asemeja al estudio de Olfert, Barr, Charlier, Greene, Zhou, y Colby, (2019) el cual tenía como objetivo mejorar los comportamientos de estilo de vida entre los estudiantes universitarios. Al término de su intervención encontraron por debajo de los rangos saludables.

El que no resultara estadísticamente significativo la eficacia del programa, no quiere decir que no haya funcionado como tal, dado que el tema de educación para la salud, es uno de los retos actuales que tienen las instituciones educativas. De acuerdo con ello Gainza-Gainza y Kerton-Manner (2015) enfatizan que para lograr el éxito y fortalecimiento en hábitos y estilos de vida saludables en estudiantes, el profesor debe tener integrado en su quehacer docente, metodológico, de investigación y orientación, el objetivo de formar ciudadanos con una cultura en salud, ya que el promover y educar con teoría y práctica por parte del docente puede impactar a mejorar la calidad de vida del individuo de forma particular y grupal preparando personas para una vida sana $\mathrm{y}$ responsables.

Por lo tanto, el programa educativo para la salud implementado en este estudio contribuye a fomentar la cultura por el bienestar físico y emocional del joven universitario. Respecto a las alternativas educativas de alimentación, actividad física y manejo del estrés y su impacto en el índice de masa corporal de la población sujeto de estudio, los resultados no demostraron un impacto estadísticamente significativo por grupo.
Pero de forma individual se puede rescatar a 29 sujetos de los cuales 16 tuvieron un decremento menor en su IMC, cuatro bajaron entre 2 y 3 kilos, y 9 se mantuvieron en su peso. Lo anterior representa el $73 \%$ de la muestra.

A partir de lo anterior los logros del programa son alentadores. Puesto, que esta es una primera etapa de la intervención. A partir de los resultados obtenidos, se pretende trabajar con la misma muestra, pero de forma individual y con un equipo interdisciplinario. Con el objetivo de abordar de manera integral la alimentación, actividad física y el estado psicológico del estudiante.

\section{Agradecimiento}

Se agradece al Instituto Tecnológico de Sonora por el financiamiento otorgado a través de PROFAPI. Con el objetivo de Fomentar, promover y apoyar el desarrollo de la investigación científica.

\section{Conclusiones}

A partir de los resultados y discusión expuesta, las conclusiones que se hacen sobre el programa de educación para la salud sobre estilos de vida y factores de riesgos en estudiantes universitarios del noroeste de México son las siguientes.

El $100 \%$ de los estudiantes presentaron tensión arterial elevada, 18 jóvenes con sobre peso y ocho con presencia de obesidad. Presencia de antecedentes hereditarios y estilos de vidas inadecuados en cuanto alimentación, manejo de estrés, sedentarismo.

Agrupando los porcentajes en cuanto al reporte de actividad física, el $84 \%$ de la muestra no realiza ejercicio según lo que se establece en la revisión de literatura. Por lo tanto, aun cuando el programa no muestra estadísticas significativas, el impacto al evaluarlo por sujeto, representa el $73 \%$ de la muestra. Esto motiva para seguir trabajando en este tipo de investigación aplicada. Dado que se diseñó el programa, se aplicó y se evaluó, esto último es generador de aprendizaje, ya que lo que no se evalúa no se puede mejorar. Y en este sentido es en lo que se pretende seguir trabajando, en corregir estrategias y procedimientos para que cada aplicación impacte a más estudiantes universitarios en edades de 18 a 20 años. 
El impartir a los estudiantes, los temas sobre las cifras de tensión arterial y como representan un riesgo para la salud; lo que significa la enfermedad de la diabetes mellitus tipos 2, el plato del buen comer, las tablas de equivalencias para una alimentación sana, la jarra del buen beber, el manejo del estrés, son un primer acercamiento para concientizar a los jóvenes sobre la importancia de hacerse responsables de su salud y que pueda generar estrategias de auto cuidado que los lleve a mejorar y suplir los malos hábitos por estilos de vidas adecuados.

Para lograr que los jóvenes estudiantes interioricen la importancia de cuidar su peso, es necesario que tengan el conocimiento conceptual, ya que este provee formación y los puede ayudar a entender la necesidad de realizar acciones por su propia salud.

El estilo de vida activo en los universitarios es un objetivo importante en la promoción de la salud, por lo que se han empezado a implementar diferentes estrategias educativas que les ayuden a desarrollarlas.

Como recomendaciones para otros investigadores que les interese trabajar con estudiantes universitarios sobre el tema de educación para la salud, se sugiere planificar sesiones cortas, aunque se prolongue el tiempo de aplicación.

Otra recomendación sería trabajarlo en forma de taller, en 4 fines de semana, para que no interfieran con las actividades de los estudiantes. Por último, diseñar programas en modalidad virtual - presencial con el fin de abarcar la mayor cantidad de estudiantes.

\section{Referencias}

Camacho, R. L., Echeverría, C. S. \& ReynosoErazo, L. (2010). Estilos de vida y riesgos en la salud de trabajadores universitarios. Journal of Behavior, Health \& Social Issues, 2(1).

Chamorro, Basilio, Y. (2010). Educación para la salud en las organizaciones escolares. Educación (10199403), 18(36), 7-19.

Gainza-Gainza y Kerton-Manner (2015). La orientación educativa en la promoción y educación para la salud en instituciones educacionales. EduSol, 15(53) págs.105-111
García, H. C., Ramos, E. D., Serrano, E. D., Sotelo, C., M., Flores, I., L. \& Reynoso-Erazo, L. (2009). Estilos de vida y riesgos a la salud en profesores universitarios: un estudio descriptivo. Psicología y Salud, 19(1), 141-149

Hernández, S. R., Fernández, C. C. y Baptista, L.P. (2016). Metodología de la investigación. McGRAW - HILL, México

Instituto Nacional de Estadística Geografía e Informática [INEGI]. (2010). Estadísticas de mortalidad por enfermedad isquémica del corazón: banco de información de INEGI. Recuperado el 10 de febrero de 2014 de http://www3.inegi.org.mx/sistemas/biinegi/ default.aspx\#T

Liang, W., Duan, Y. P., Shang, B. R., Wang, Y. P., Hu, C., \& Lippke, S. (2019). A web-based lifestyle intervention program for Chinese college students: study protocol and baseline characteristics of a randomized placebocontrolled trial. BMC public health, 19(1), 1-11

Olfert, M. D., Barr, M. L., Charlier, C. C., Greene, G. W., Zhou, W., \& Colby, S. E. (2019). Sex differences in lifestyle behaviors among US college freshmen. International journal of environmental research and public health, 16(3), 482.

Pancich Flores, I.A., Ángel Toledo, E., Monroy, M y Reynoso-Erazo, L. (2011). Estilo de vida e hipertensión en jóvenes universitarios. Presentado en el $5^{\circ}$ Congreso Latinoamericano de Psicología de la Salud, en Xalapa, Ver., del 18 al 20 de mayo de 2011.

Organización Mundial de la Salud (2013). 10 datos sobre las enfermedades no transmisibles. Recuperado el 5 de agosto del 2019 de: http://www.who.int/features/factfiles/noncomm unicable_diseases/es/

Organización Mundial de la salud (2015). Obesidad y sobrepeso. Recuperado el 5 de abril del 2019 de: http://www.who.int/mediacentre/factsheets/fs 31 1/es/

Organización Mundial de la Salud (2018). Vida saludable, bienestar y objetivos de desarrollo sostenible. Recuperado el 03 de abril del 2019 de Bulletin of the World Health Organization 2018;96:590-590A. doi: http://dx.doi.org/10.2471/BLT.18.222042

BOJORQUEZ-DIAZ, Cecilia Ivonne, REYNOSO-ERAZO, Leonardo, FAVELA-RAMIREZ, Carlos Artemio y CASTRO-ROBLES, Alejandra Isabel. Programa de educación para la salud: Estilos de vida y factores de riesgos en universitarios. Revista de Gestión Universitaria. 2019 
Whatnall, M. C., Patterson, A. J., Brookman, S., Convery, P., Swan, C., Pease, S., \&

Hutchesson, M. J. (2019). Lifestyle behaviors and related health risk factors in a sample of Australian university students. Journal of American College Health, 1-8.

Wolferz Jr, R., Arjani, S., Bolze, A., \& Frates, E. P. (2019). Students Teaching Students:

Bringing Lifestyle Medicine Education to Middle and High Schools Through Student -Led Community Outreach Programs. American Journal of Lifestyle Medicine, 1559827619836970. 